\title{
Measuring consensus in a preference-approval context
}

\author{
Bora Erdamar ${ }^{\text {a }}$, José Luis García-Lapresta ${ }^{\mathrm{b}}$, \\ David Pérez-Román ${ }^{\mathrm{b}}$, M. Remzi Sanver ${ }^{\mathrm{c}}$ \\ ${ }^{a}$ Institute of Social Sciences, Istanbul Bilgi University, Turkey. \\ ${ }^{\mathrm{b}}$ PRESAD Research Group, University of Valladolid, Spain. \\ ${ }^{\mathrm{c}}$ Murat Sertel Center for Advanced Economic Studies, Istanbul Bilgi University, \\ Turkey.
}

\begin{abstract}
We consider measuring the degree of homogeneity for preference-approval profiles which include the approval information for the alternatives as well as the rankings of them. A distance-based approach is followed to measure the disagreement for any given two preference-approvals. Under the condition that a proper metric is used, we propose a measure of consensus which is robust to some extensions of the ordinal framework. This paper also shows that there exists a limit for increasing the homogeneity level in a group of individuals by simply replicating their preferenceapprovals.
\end{abstract}

Key words: Consensus, Approval voting, Preference-approval, Kemeny metric, Hamming metric.

\section{Introduction}

In collective decision making problems, the notion of consensus has been analyzed and interpreted in miscellaneous ways. The dictionary meaning of consensus is a general (unanimous) agreement within a group of people or agents. However, most of the decision making procedures (e.g. elections, voting by

Email addresses: berdamar@gmail.com (Bora Erdamar), lapresta@eco.uva.es (José Luis García-Lapresta), david@emp.uva.es (David Pérez-Román), sanver@bilgi.edu.tr (M. Remzi Sanver). 
committees, competitions) deal with a more realistic situation of partial agreement for the candidates or alternatives. Interpreting a partial agreement of individuals as a consensus up to some degree, the immediate question is how to measure that degree of agreement (Kacprzyk [1], Tastle and Wierman [2,3]). Related questions include how to use this information to reach a final decision (Kemeny [4], Beliakov, Calvo and James [5]) and which procedures can be used to increase the level of consensus (Susskind and McKearnan [6], Strauss and Layton [7], Van Den Belt [8]). For an overview of different attributions of consensus, one can also see Martínez-Panero [9]. In this paper, consensus is interpreted as the degree of homogeneity within a set of individuals and consensus measure is a scale for the similarity of preferences.

It is important to note that the degree of consensus depends on the context of preferences. Similarity of preferences when individuals submit linear orders over alternatives can be very different from the homogeneity of a profile composed of weak orders. In the related literature, Kendall and Gibbons [10] considered measuring concordance among only two linear orders. Then, Hays [11] and Alcalde-Unzu and Vorsatz [12] generalized the idea to any number of linear orders. Similarly, Bosch [13] proposed a measure of consensus for any given profile of linear orders by a mapping which assigns a number between 0 and 1 according to the degree of homogeneity in that profile. Satisfying some desirable axioms such as unanimity (for every subgroup of agents, the highest degree of consensus is reached only if all agents have the same orderings), anonymity (permutation of agents does not lead to a change in the degree of consensus) and neutrality (permutation of alternatives does not lead to a change in the degree of consensus) Bosch's model has been investigated further for various domains. García-Lapresta and Pérez-Román [14] extended the consensus measure of Bosch [13] for weak orders and introduced new properties such as maximum dissension (in each subset of two agents, the minimum consensus is reached only if agents have linear orders which are inverses of each other) and reciprocity (replacing each order in the profile by their inverses does not lead to a change in the degree of consensus). Moreover, GarcíaLapresta and Pérez-Román [15] further extended the framework of Bosch [13] for weighted Kemeny distances, thereby dealing with the possibility of weighting discrepancies among weak orders.

Some recent models for collective decision making problems (e.g. approval voting [16], majority judgment [17], range voting [18]) use non-standard formulations of inputs in aggregation of preferences. These models assume that individuals adopt a common language when they evaluate alternatives. Therefore, instead of aggregating ordinal rankings these models deal with aggregating labels such as approved and disapproved. Brams and Sanver [19] suggest a framework that can be considered as a compromise between standard and non-standard models by combining the information of ranking and approval in a hybrid system which they call preference-approval. Individuals are assumed 
to submit a weak ordering on a given set of alternatives and a cut-off line to distinguish acceptable and unacceptable alternatives for them. An alternative which is ranked above (resp. below) the line is qualified as acceptable (resp. unacceptable). Preference-approval model extends the ordinal framework in a minimal way by incorporating two qualifications good and bad with a common meaning among individuals. It is worthwhile to note that the status-quo point in bargaining problems, the threshold level in public good problems and the alternative of being self-matched in matching problems can be interpreted as the cut-off lines when these models are translated to the preference-approvals. In that sense, preference-approval model proposes a common framework in which non-standard aggregation procedures and the standard ones in the literature can be analyzed by a natural way.

The problem of how to measure consensus for the extended ordinal frameworks is an open question in the literature. In this contribution, by following a distance-based approach we focus on measuring the degree of disagreement/agreement in preference-approval profiles. Since distance functions widely used in the literature are defined on various domains of ordinal rankings, the first difficulty is to derive a proper metric for extensions of weak orders. We propose a way of measuring distance separately for the two types of informational content in preference-approvals and then we derive a metric defined by a convex combination of these distances. Technically speaking, for any given pair of preference-approvals, first we use Kemeny metric [4] for weak orders to measure the distance regarding the ranking information. Secondly, we use Hamming metric [20] to measure the concordance with respect to the acceptable or unacceptable alternatives. Proper aggregation of these two types of distances depends on the context of the particular problem. Noting that the choice of a particular convex combination of Kemeny and Hamming distances reflects the emphasis on the disagreement regarding approval or ranking, we briefly discuss various ways for aggregation. Then, we propose a measure of consensus (based on García-Lapresta and Pérez-Román [14]) which is shown to be robust to some extensions of the ordinal framework under the condition that a proper metric is used. By investigating the properties of the consensus measure for preference-approvals, we also show a surprising result that the degree of homogeneity in a group of individuals cannot be increased by simply replicating their preferences in this model.

This paper is organized as follows. In section 2, the basic notation and notions are introduced. Section 3 is devoted to the definitions and some properties of consensus measures in general. Section 4 includes our proposal for measuring consensus in preference-approval context and some results. Finally, in section 5 concluding remarks are made including some possible further extensions of the model. 


\section{Preliminaries}

Consider a set of agents $V=\left\{v_{1}, \ldots, v_{m}\right\}$ with $m \geq 2$ confronting a finite set of alternatives $X=\left\{x_{1}, \ldots, x_{n}\right\}$ where $n \geq 2$. We assume that each agent ranks the alternatives in $X$ by means of a weak order and additionally, evaluates each alternative as either acceptable or unacceptable by partitioning the alternative set into approved (good) and disapproved (bad) alternatives. These two types of information exhibit the following consistency: given two alternatives $x$ and $y$, if $x$ is approved and $y$ is disapproved, then $x$ is ranked above $y$.

Technically speaking, by a weak order (or complete preorder) on $X$ we mean a complete $^{1}$ and transitive binary relation on $X$.

On the other hand, a linear order on $X$ is an antisymmetric weak order on $X$. We write $W(X)$ for the set of weak orders on $X$ and $L(X)$ for the set of linear orders on $X$.

Given $R \in W(X)$, we let $\succ$ and $\sim$ stand for the asymmetric and the symmetric parts of $R$, respectively, i.e.,

$$
\begin{aligned}
x_{i} & \succ x_{j} \Leftrightarrow \operatorname{not}\left(x_{j} R x_{i}\right) \\
x_{i} & \sim x_{j} \Leftrightarrow\left(x_{i} R x_{j} \text { and } x_{j} R x_{i}\right) .
\end{aligned}
$$

By $\mathcal{P}(V)$ we denote the power set of $V$, i.e., $I \in \mathcal{P}(V) \Leftrightarrow I \subseteq V$; and by $\mathcal{P}_{2}(V)$ we mean the collection of subsets of $V$ with at least two elements. That is, $\mathcal{P}_{2}(V)=\{I \in \mathcal{P}(V) \mid \# I \geq 2\}$, where $\# I$ is the cardinality of $I$. Analogously, we write $\mathcal{P}(X)$ for the power set of $X$.

Finally, we denote $\boldsymbol{a}=\left(a_{1}, \ldots, a_{n}\right)$ for the vectors in $\mathbb{R}^{n}$.

\subsection{Preference-approval structures}

For any given set of $X$ of alternatives, we define preference-approvals by partitioning $X$ into $A$ the set of acceptable (or good) alternatives and $U=X \backslash A$ the set of unacceptable (or bad) alternatives, where $A$ and $U$ can be empty sets.

$\overline{1}$ By completeness, for any given $x_{i}$ and $x_{j}$ in $X$, either $x_{i}$ is at least as good as $x_{j}$ or $x_{j}$ is at least as good as $x_{i}$. Hence, any complete binary relation is also reflexive. 
Definition $1 A$ preference-approval on $X$ is a pair $(R, A) \in W(X) \times \mathcal{P}(X)$ satisfying the following condition

$$
\forall x_{i}, x_{j} \in X\left(\left(x_{i} R x_{j} \text { and } x_{j} \in A\right) \Rightarrow x_{i} \in A\right) .
$$

Note that if $x_{i} R x_{j}$ and $x_{i} \in U$, then we have $x_{j} \in U$.

We denote $\mathcal{R}(X)$ for the set of preference-approvals on $X$.

Given $R \in W(X)$, we let $R^{-1}$ be the inverse of $R$ such that

$$
x_{i} R^{-1} x_{j} \Leftrightarrow x_{j} R x_{i}
$$

for all $x_{i}, x_{j} \in X$. Similarly, given a preference-approval $(R, A) \in \mathcal{R}(X)$, we write $(R, A)^{-1}=\left(R^{-1}, X \backslash A\right)$ for the preference-approval which is the inverse of $(R, A)$.

Example 1 In order to illustrate preference-approval structures, consider the following example:

$$
\begin{gathered}
x_{2} x_{3} x_{5} \\
x_{1} \\
\hline x_{4} x_{7} \\
x_{6}
\end{gathered}
$$

where alternatives in the same row are indifferent, alternatives in upper rows are preferred to those located in lower rows, alternatives above the dash line are acceptable (good) and those below the dash line are unacceptable (bad).

The inverse of the preference-approval above is the following:

$$
\begin{gathered}
x_{6} \\
x_{4} x_{7} \\
\hline x_{1} \\
x_{2} x_{3} x_{5}
\end{gathered}
$$

We now introduce a system for codifying each preference-approval structure $(R, A) \in \mathcal{R}(X)$ by means of two vectors: $\boldsymbol{p}_{R} \in \mathbb{R}^{n}$ that represents the position of the alternatives, and $\boldsymbol{i}_{A} \in\{0,1\}^{n}$ that represents acceptable alternatives.

It is worthwhile to note that there does not exist a unique system for codifying weak orders, since a weak order can be linearized in many different ways. We propose a codification based on a linearization of the weak order by assigning 
each alternative the average of the positions of the alternatives within the same equivalence class.

Following García-Lapresta and Pérez-Román [14], for any given $R \in W(X)$ we assign the position of each alternative $x_{j}$ in $R$ through the mapping $P_{R}: X \longrightarrow \mathbb{R}$ defined as

$$
P_{R}\left(x_{j}\right)=n-\#\left\{x_{i} \in X \mid x_{j} \succ x_{i}\right\}-\frac{1}{2} \#\left\{x_{i} \in X \backslash\left\{x_{j}\right\} \mid x_{i} \sim x_{j}\right\},
$$

where $n$ is the number of alternatives.

The following table illustrates the codification of the preference-approval in Example 1.

$\begin{array}{r}\hline P_{R}\left(x_{1}\right)=7-3-\frac{1}{2} \cdot 0=4 \\ \hline P_{R}\left(x_{2}\right)=7-4-\frac{1}{2} \cdot 2=2 \\ \hline P_{R}\left(x_{3}\right)=7-4-\frac{1}{2} \cdot 2=2 \\ \hline P_{R}\left(x_{4}\right)=7-1-\frac{1}{2} \cdot 1=5.5 \\ \hline P_{R}\left(x_{5}\right)=7-4-\frac{1}{2} \cdot 2=2 \\ \hline P_{R}\left(x_{6}\right)=7-0-\frac{1}{2} \cdot 0=7 \\ \hline P_{R}\left(x_{7}\right)=7-1-\frac{1}{2} \cdot 1=5.5 \\ \hline\end{array}$

We denote $\boldsymbol{p}_{R}=\left(P_{R}\left(x_{1}\right), \ldots, P_{R}\left(x_{n}\right)\right)$ for the position vector of $R \in W(X)$. Note that the codification vector in Example 1 is $\boldsymbol{p}_{R}=(4,2,2,5.5,2,7,5.5)$.

On the other hand, given $A \subseteq X$, we define $I_{A}: X \longrightarrow\{0,1\}$ the indicator function (or characteristic function) of $A$ :

$$
I_{A}\left(x_{j}\right)=\left\{\begin{array}{l}
1, \text { if } x_{j} \in A, \\
0, \text { if } x_{j} \in X \backslash A .
\end{array}\right.
$$

By $\boldsymbol{i}_{A}=\left(I_{A}\left(x_{1}\right), \ldots, I_{A}\left(x_{n}\right)\right)$ we denote the indicator vector of $A \subseteq X$.

Note that the preference-approval in Example 1 will be codified as $\boldsymbol{i}_{A}=$ $(1,1,1,0,1,0,0)$ since $x_{1}, x_{2}, x_{3}, x_{5}$ are the accepted alternatives and $x_{4}, x_{6}$ and $x_{7}$ are the unaccepted ones.

Given a preference-approval $(R, A)$, we can completely characterize it by the $\left(\boldsymbol{p}_{R}, \boldsymbol{i}_{A}\right)$ tuple. 
Remark 1 The condition appearing in Definition 1 can be written as:

$$
\left(P_{R}\left(x_{i}\right) \geq P_{R}\left(x_{j}\right) \text { and } I_{A}\left(x_{j}\right)=1\right) \Rightarrow I_{A}\left(x_{i}\right)=1 .
$$

\subsection{Distances and metrics}

Usually, distance and metric are considered as synonymous. However, we follow the approach given by Deza and Deza [21], where distances and metrics are different concepts.

Definition $2 A$ distance on a set $D \neq \emptyset$ is a mapping $d: D \times D \longrightarrow \mathbb{R}$ satisfying the following conditions for all $a, b \in A$ :

(1) $d(a, b) \geq 0$ (non-negativity),

(2) $d(a, b)=d(b, a)$ (symmetry),

(3) $d(a, a)=0$ (reflexivity).

If $d$ satisfies the following additional conditions for all $a, b, c \in A$ :

(4) $d(a, b)=0 \Leftrightarrow a=b$ (identity of indiscernibles),

(5) $d(a, b) \leq d(a, c)+d(c, b)$ (triangle inequality),

then we say that $d$ is a metric.

We now focus on Kemeny and Hamming metrics. Since any preference-approval has two components, an ordering and a partition on the set of alternatives, calculating the distance between any two preference-approvals requires to measure distances with respect to these components. We propose using Kemeny metric for weak orders and Hamming metric for the information regarding acceptable alternatives.

\subsubsection{The Kemeny metric}

The Kemeny metric was initially defined on linear orders by Kemeny [4], as the sum of pairs where the ranking of these pairs are different in the linear orders. Subsequently, it has been generalized to the framework of weak orders (see Cook, Kress and Seiford [22] and Eckert and Klamler [23], among others).

Typically, the Kemeny metric on weak orders $d_{K}: W(X) \times W(X) \longrightarrow \mathbb{R}$ is defined as the cardinality of the symmetric difference between the weak orders, i.e.,

$$
d_{K}\left(R_{1}, R_{2}\right)=\#\left(\left(R_{1} \cup R_{2}\right) \backslash\left(R_{1} \cap R_{2}\right)\right) .
$$

In this paper, having a codification based approach we adopt the definition 
of Kemeny metric proposed by García-Lapresta and Pérez-Román [14] as the following:

$$
d_{K}\left(R_{1}, R_{2}\right)=\sum_{\substack{i, j=1 \\ i<j}}^{n}\left|\operatorname{sgn}\left(P_{R_{1}}\left(x_{i}\right)-P_{R_{1}}\left(x_{j}\right)\right)-\operatorname{sgn}\left(P_{R_{2}}\left(x_{i}\right)-P_{R_{2}}\left(x_{j}\right)\right)\right|,
$$

where sgn is the sign function:

$$
\operatorname{sgn}(a)=\left\{\begin{aligned}
1, & \text { if } a>0, \\
0, & \text { if } a=0, \\
-1, & \text { if } a<0
\end{aligned}\right.
$$

It is worthwhile to remark that the Kemeny metric is a bounded metric in $W(X)$. That is, there exists some $M>0$ such that $d_{K}\left(R_{1}, R_{2}\right) \leq M$ for all $R_{1}, R_{2} \in W(X)$. One can immediately check that the maximum distance between orders with respect to Kemeny metric is $(\# X)^{2}-\# X$.

\subsubsection{The Hamming metric}

The Hamming metric (Hamming [20]) $d_{H}: \mathbb{R}^{n} \times \mathbb{R}^{n} \longrightarrow \mathbb{R}$ is defined as ${ }^{2}$

$$
d_{H}(\boldsymbol{a}, \boldsymbol{b})=\#\left\{i \in\{1, \ldots, n\} \mid a_{i} \neq b_{i}\right\} .
$$

We extend the Hamming metric from $\mathbb{R}^{n}$ to $\mathcal{P}(X)$ as the mapping $d_{H}: \mathcal{P}(X) \times \mathcal{P}(X) \longrightarrow \mathbb{R}$ defined by

$$
d_{H}\left(A_{1}, A_{2}\right)=d_{H}\left(\boldsymbol{i}_{A_{1}}, \boldsymbol{i}_{A_{2}}\right) .
$$

Note that the Hamming metric formulation above is equivalent to the following one.

$$
d_{H}\left(A_{1}, A_{2}\right)=\#\left(\left(A_{1} \cup A_{2}\right) \backslash\left(A_{1} \cap A_{2}\right)\right) .
$$

Clearly, the Hamming metric on $\mathcal{P}(X)$ is a bounded metric as well and one can easily check that the maximum distance between any two subsets of $X$ is $\# X$.

$\overline{2}$ On binary vectors $\boldsymbol{a}, \boldsymbol{b} \in\{0,1\}^{n}$, the Hamming metric and the $l_{1}$-metric (or Manhattan metric) coincide:

$$
d_{H}(\boldsymbol{a}, \boldsymbol{b})=\sum_{i=1}^{n}\left|a_{i}-b_{i}\right|
$$




\subsubsection{Mixing distances and metrics}

In what follows, we state that $d_{K}$ and $d_{H}$, although measuring distances regarding different kinds of information separately, cannot be aggregated as a total distance since $d_{K}$ and $d_{H}$ do not have the same codomains. Therefore, we first normalize these two metrics to the same codomain $[0,1]$ via dividing by their maximum distances and we get $d_{R}$ and $d_{A}$ as distances regarding orderings and acceptable alternatives, respectively.

\section{Definition 3}

(1) The mapping $d_{R}: \mathcal{R}(X) \times \mathcal{R}(X) \longrightarrow[0,1]$ is defined as

$$
d_{R}\left(\left(R_{1}, A_{1}\right),\left(R_{2}, A_{2}\right)\right)=\frac{d_{K}\left(R_{1}, R_{2}\right)}{(\# X)^{2}-\# X}=\frac{\#\left(\left(R_{1} \cup R_{2}\right) \backslash\left(R_{1} \cap R_{2}\right)\right)}{(\# X)^{2}-\# X} .
$$

(2) The mapping $d_{A}: \mathcal{R}(X) \times \mathcal{R}(X) \longrightarrow[0,1]$ is defined as

$$
d_{A}\left(\left(R_{1}, A_{1}\right),\left(R_{2}, A_{2}\right)\right)=\frac{d_{H}\left(A_{1}, A_{2}\right)}{\# X}=\frac{\#\left(\left(A_{1} \cup A_{2}\right) \backslash\left(A_{1} \cap A_{2}\right)\right)}{\# X} .
$$

\section{Proposition 1}

(1) $d_{R}$ is a distance on $\mathcal{R}(X)$ and for all $\left(R_{1}, A_{1}\right),\left(R_{2}, A_{2}\right) \in \mathcal{R}(X)$ it holds

(a) $d_{R}\left(\left(R_{1}, A_{1}\right),\left(R_{2}, A_{2}\right)\right)=0 \Leftrightarrow R_{1}=R_{2}$.

(b) $d_{R}$ verifies triangle inequality.

(c) $d_{R}\left(\left(R_{1}, A_{1}\right),\left(R_{2}, A_{2}\right)\right)=1 \Leftrightarrow\left(R_{1}, R_{2} \in L(X)\right.$ and $\left.R_{2}=R_{1}^{-1}\right)$.

(2) $d_{A}$ is a distance on $\mathcal{R}(X)$ and for all $\left(R_{1}, A_{1}\right),\left(R_{2}, A_{2}\right) \in \mathcal{R}(X)$ it holds

(a) $d_{A}\left(\left(R_{1}, A_{1}\right),\left(R_{2}, A_{2}\right)\right)=0 \Leftrightarrow A_{1}=A_{2}$.

(b) $d_{A}$ verifies triangle inequality.

(c) $d_{A}\left(\left(R_{1}, A_{1}\right),\left(R_{2}, A_{2}\right)\right)=1 \Leftrightarrow A_{2}=X \backslash A_{1}$.

(3) Neither $d_{R}$ nor $d_{A}$ are metrics on $\mathcal{R}(X)$.

Proof: Let $\left(R_{1}, A_{1}\right),\left(R_{2}, A_{2}\right) \in \mathcal{R}(X)$.

(1) Since $d_{R}$ is the Kemeny metric normalized by a number, the properties of non-negativity, symmetry and reflexivity are obvious.

(a) $d_{R}\left(\left(R_{1}, A_{1}\right),\left(R_{2}, A_{2}\right)\right)=0 \Leftrightarrow d_{K}\left(R_{1}, R_{2}\right)=0 \Leftrightarrow R_{1}=R_{2}$.

(b) $d_{R}$ inherits from Kemeny metric the property of triangle inequality.

(c) In García-Lapresta and Pérez-Román [14], it is proven that for the Kemeny metric, the maximum distance between weak orders is not reached when one of them is not linear and additionally, the maximum distance between linear orders is not reached when they are not inverses of each other. 
(2) Since $d_{A}$ is the Hamming metric normalized by a number, non-negativity, symmetry and reflexivity are obvious.

(a) $d_{A}\left(\left(R_{1}, A_{1}\right),\left(R_{2}, A_{2}\right)\right)=0 \Leftrightarrow d_{H}\left(A_{1}, A_{2}\right)=0 \Leftrightarrow A_{1}=A_{2}$.

(b) $d_{A}$ inherits the property of triangle inequality from the Hamming metric .

(c) $d_{A}\left(\left(R_{1}, A_{1}\right),\left(R_{2}, A_{2}\right)\right)=1 \Leftrightarrow\left(A_{1} \cup A_{2}=X\right.$ and $\left.A_{1} \cap A_{2}=\emptyset\right)$, i.e., $A_{2}=X \backslash A_{1}$.

(3) Let $\left(R_{1}, A_{1}\right),\left(R_{2}, A_{2}\right) \in \mathcal{R}(X)$ be such that $R_{1} \neq R_{2}$ and $A_{1} \neq A_{2}$. Then, we have $d_{R}\left(\left(R_{1}, A_{1}\right),\left(R_{1}, A_{2}\right)\right)=d_{A}\left(\left(R_{1}, A_{1}\right),\left(R_{2}, A_{1}\right)\right)=0$. Consequently, $d_{R}$ and $d_{A}$ do not verify identity of indiscernibles, hence they are not metrics.

The following example illustrates the calculation of distances for a given profile.

Example 2 Consider four agents confronting a set of four alternatives $X=$ $\left\{x_{1}, x_{2}, x_{3}, x_{4}\right\}$ and having the following preference-approvals:

\begin{tabular}{|c|c|c|c|}
\hline$\left(R_{1}, A_{1}\right)$ & $\left(R_{2}, A_{2}\right)$ & $\left(R_{3}, A_{3}\right)$ & $\left(R_{4}, A_{4}\right)$ \\
\hline$x_{1}$ & $x_{2}$ & $x_{1}$ & $x_{3}$ \\
\hline$x_{2}$ & $x_{1}$ & $x_{2}$ & $x_{2}$ \\
\hline$x_{3} x_{4}$ & $x_{3} x_{4}$ & $x_{3} x_{4}$ & $x_{1} x_{4}$ \\
\hline
\end{tabular}

These preference-approvals are codified as follows:

$$
\begin{array}{ll}
\boldsymbol{p}_{R_{1}}=(1,2,3.5,3.5) & \boldsymbol{i}_{A_{1}}=(1,1,0,0) \\
\boldsymbol{p}_{R_{2}}=(2,1,3.5,3.5) & \boldsymbol{i}_{A_{2}}=(1,1,0,0) \\
\boldsymbol{p}_{R_{3}}=(1,2,3.5,3.5) & \boldsymbol{i}_{A_{3}}=(1,0,0,0) \\
\boldsymbol{p}_{R_{4}}=(3.5,2,1,3.5) & \boldsymbol{i}_{A_{4}}=(0,0,0,0)
\end{array}
$$

The following table shows the distances $d_{R}$ and $d_{A}$ between preferenceapprovals: 


\begin{tabular}{c|c|c|} 
& $d_{R}$ & $d_{A}$ \\
\hline$\left(R_{1}, A_{1}\right),\left(R_{2}, A_{2}\right)$ & $\frac{2}{12}$ & 0 \\
\hline$\left(R_{1}, A_{1}\right),\left(R_{3}, A_{3}\right)$ & 0 & $\frac{3}{12}$ \\
\hline$\left(R_{1}, A_{1}\right),\left(R_{4}, A_{4}\right)$ & $\frac{8}{12}$ & $\frac{6}{12}$ \\
\hline$\left(R_{2}, A_{2}\right),\left(R_{3}, A_{3}\right)$ & $\frac{2}{12}$ & $\frac{3}{12}$ \\
\hline$\left(R_{2}, A_{2}\right),\left(R_{4}, A_{4}\right)$ & $\frac{6}{12}$ & $\frac{6}{12}$ \\
\hline$\left(R_{3}, A_{3}\right),\left(R_{4}, A_{4}\right)$ & $\frac{8}{12}$ & $\frac{3}{12}$ \\
\hline
\end{tabular}

Note that the minimum distance regarding orderings is in between $\left(R_{1}, A_{1}\right)$ and $\left(R_{2}, A_{2}\right)$ since there is a disagreement only on the ranking of the first two alternatives. On the other hand, the maximum distance regarding orderings in this profile is attained by $\left(R_{4}, A_{4}\right)$ and $\left(R_{1}, A_{1}\right)$, which is also the distance between $\left(R_{4}, A_{4}\right)$ and $\left(R_{3}, A_{3}\right)$. Note that, for these tuples there is only one pair of alternatives (namely $\left(x_{2}, x_{4}\right)$ ) for which these preference-approvals agree on.

Similarly, the minimum distance regarding acceptability is in between $\left(R_{1}, A_{1}\right)$ and $\left(R_{2}, A_{2}\right)$ since there is a full agreement for the set of acceptable and unacceptable alternatives. On the other hand, the maximum distance regarding acceptability is attained by $\left(R_{4}, A_{4}\right)$ and $\left(R_{1}, A_{1}\right)$ which is also the distance between $\left(R_{4}, A_{4}\right)$ and $\left(R_{2}, A_{2}\right)$. Note that there is a disagreement on the acceptability of two alternatives (namely $x_{1}$ and $x_{2}$ ) for these preference-approvals.

For the rest of the section, first we define the neutrality of metrics and then we establish that a neutral metric can be deduced from the convex combinations of $d_{R}$ and $d_{A}$.

Definition 4 A set $D \subseteq \mathbb{R}^{n}$ is stable under permutations if for every permutation $\sigma$ on $\{1, \ldots, n\}$, it holds $\left(a_{1}^{\sigma}, \ldots, a_{n}^{\sigma}\right) \in D$ for every $\left(a_{1}, \ldots, a_{n}\right) \in D$.

Definition 5 Given a set $D \subseteq \mathbb{R}^{n}$ stable under permutations, a distance (or metric) $d: D \times D \longrightarrow \mathbb{R}$ is neutral if for every permutation $\sigma$ on $\{1, \ldots, n\}$ it holds

$$
d\left(\left(a_{1}^{\sigma}, \ldots, a_{n}^{\sigma}\right),\left(b_{1}^{\sigma}, \ldots, b_{n}^{\sigma}\right)\right)=d\left(\left(a_{1}, \ldots, a_{n}\right),\left(b_{1}, \ldots, b_{n}\right)\right),
$$

for all $\left(a_{1}, \ldots, a_{n}\right),\left(b_{1}, \ldots, b_{n}\right) \in D$.

Remark 2 The Kemeny metric is neutral (see García-Lapresta and PérezRomán [14]). One can easily check that the Hamming metric is neutral as well.

Remark 3 Given two distances $d_{1}, d_{2}: D \times D \longrightarrow \mathbb{R}$, for every $\lambda \in[0,1]$ 
the convex combination $\lambda d_{1}+(1-\lambda) d_{2}$ is also a distance.

In the next result we show that although $d_{R}$ and $d_{A}$ are not metrics, their convex combinations are always metrics except for the degenerate values of $\lambda=0$ and $\lambda=1$.

Proposition 2 For every $\lambda \in(0,1)$ and all $\left(R_{1}, A_{1}\right),\left(R_{2}, A_{2}\right) \in \mathcal{R}(X)$, the following statements hold:

(1) $d_{\lambda}=\lambda d_{R}+(1-\lambda) d_{A}$ is a neutral metric and $d_{\lambda}\left(\left(R_{1}, A_{1}\right),\left(R_{2}, A_{2}\right)\right) \leq 1$.

(2) $d_{\lambda}\left(\left(R_{1}, A_{1}\right),\left(R_{2}, A_{2}\right)\right)=1$ if and only if $R_{1}, R_{2} \in L(X), R_{2}=R_{1}^{-1}$ and $A_{2}=X \backslash A_{1}$.

PROOF:

(1) By Remark $3, d_{\lambda}$ is a distance. By Proposition $1, d_{\lambda}$ verifies the identity of indiscernibles property and the triangle inequality. Then, $d_{\lambda}$ is a metric. By Remark 2, the Kemeny and Hamming metrics are neutral and it is obvious that the convex of combination $\lambda d_{R}+(1-\lambda) d_{A}$ satisfies neutrality, too.

(2) By Proposition 1.

It is worthwhile to note that the aggregation of two distances for different kinds of information leads to two problems. The first one, which is technical, arises from the fact that $d_{K}$ and $d_{H}$ have different codomains for aggregation and a solution to this problem has been proposed by the proposition 2 . The second one is deciding on the appropriate value of $\lambda$ for the aggregation of these two distances. Since $\lambda$ (resp. $1-\lambda$ ) determines the weight of information regarding orderings (resp. acceptability), the value of $\lambda$ should be decided before the implementation of the consensus measuring.

In practice, the selection of the lambda can be done in various ways. First, as in the case of voting in the committees, a moderator or a decision maker can decide on $\lambda$ according to his principles. Although $\lambda$ can take infinitely many values, the most important decision would be choosing the component of the preference (orderings or approval) that will have more weight than the other. Second, a separate aggregation rule can be applied and the outcome of that rule can be used as an optimal value of the $\lambda$. In particular, the mean or a trimmed mean of the submitted $\lambda$ values can be used as the outcome. However, when an aggregation procedure is followed the issues regarding strategic behavior should be taken into consideration.

Example 3 The following table illustrates the changes in the total distances between preference-approvals in Example 2 with respect to the values of $\lambda$. 


\begin{tabular}{llll} 
& $\lambda=0.25$ & $\lambda=0.5$ & $\lambda=0.75$ \\
\hline$d_{\lambda}\left(\left(R_{1}, A_{1}\right),\left(R_{2}, A_{2}\right)\right)$ & 0.04167 & 0.08333 & 0.125 \\
\hline$d_{\lambda}\left(\left(R_{1}, A_{1}\right),\left(R_{3}, A_{3}\right)\right)$ & 0.1875 & 0.125 & 0.0625 \\
\hline$d_{\lambda}\left(\left(R_{1}, A_{1}\right),\left(R_{4}, A_{4}\right)\right)$ & 0.54267 & 0.58333 & 0.625 \\
\hline$d_{\lambda}\left(\left(R_{2}, A_{2}\right),\left(R_{3}, A_{3}\right)\right)$ & 0.22917 & 0.20833 & 0.1875 \\
\hline$d_{\lambda}\left(\left(R_{2}, A_{2}\right),\left(R_{4}, A_{4}\right)\right)$ & 0.5 & 0.5 & 0.5 \\
\hline$d_{\lambda}\left(\left(R_{3}, A_{3}\right),\left(R_{4}, A_{4}\right)\right)$ & 0.35417 & 0.4583 & 0.5625 \\
\hline
\end{tabular}

In these results, note that the preference-approval which has the minimum distance to $\left(R_{1}, A_{1}\right)$ is $\left(R_{2}, A_{2}\right)$ when we have $\lambda=0.25$. However, when $\lambda=0.75$ the result changes to $\left(R_{3}, A_{3}\right)$. To see why, note that when $\lambda=0.25$ the distance $d_{A}$ is weighted more than $d_{R}$ implying that the disagreement on the set of accepted alternatives is more important than the disagreement on the orderings. This is reversed when $\lambda=0.75$. For another illustration of a similar change in the distances with respect to $\lambda$, check that among the given preference-approvals $\left(R_{3}, A_{3}\right)$ is the closest to $\left(R_{4}, A_{4}\right)$ for $\lambda=0.25$. However, for $\lambda=0.75$ the previous result changes to $\left(R_{2}, A_{2}\right)$.

\section{Consensus measures}

Consensus measures have been introduced and analyzed by Bosch [13] in the context of linear orders. Subsequently, García-Lapresta and Pérez-Román $[14,24]$ extended this notion to the context of weak orders by using distances. Although many non-standard preferences are also analyzed for aggregation problems, the problem of measuring homogeneity for a set of these non-standard preferences are not fully investigated in the literature. In this section, we focus on consensus measures for preference-approval structures and start introducing basic notions for consensus measures in general.

\subsection{Basic notions}

First, some pieces of notation are included.

Definition $6 A$ profile is a vector $\boldsymbol{R}=\left(\left(R_{1}, A_{1}\right), \ldots,\left(R_{m}, A_{m}\right)\right) \in \mathcal{R}(X)^{m}$ of preference-approvals, where $\left(R_{i}, A_{i}\right)$ contains the preference-approval of the agent $v_{i}$, with $i=1, \ldots, m$.

(1) The inverse of $\boldsymbol{R}$ is $\boldsymbol{R}^{-1}=\left(\left(R_{1}^{-1}, X \backslash A_{1}\right), \ldots,\left(R_{m}^{-1}, X \backslash A_{m}\right)\right)$. 
(2) Given a permutation $\pi$ on $\{1, \ldots, m\}$ and $\emptyset \neq I \subseteq V$, we denote $\boldsymbol{R}_{\pi}=\left(\left(R_{\pi(1)}, A_{\pi(1)}\right), \ldots,\left(R_{\pi(m)}, A_{\pi(m)}\right)\right)$ and $I_{\pi}=\left\{v_{\pi^{-1}(i)} \mid v_{i} \in I\right\}$, i.e., $v_{j} \in I_{\pi} \Leftrightarrow v_{\pi(j)} \in I$.

(3) Given a permutation $\sigma$ on $\{1, \ldots, n\}$, we denote by $\boldsymbol{R}^{\sigma}=\left(\left(R_{1}^{\sigma}, A_{1}^{\sigma}\right), \ldots,\left(R_{m}^{\sigma}, A_{m}^{\sigma}\right)\right)$ the profile obtained from $\boldsymbol{R}$ by relabeling the alternatives according to $\sigma$, i.e., $x_{i} R_{k} x_{j} \Leftrightarrow x_{\sigma(i)} R_{k}^{\sigma} x_{\sigma(j)}$ and $x_{i} \in A_{k}^{\sigma} \Leftrightarrow x_{\sigma(i)} \in A_{k}$, for all $i, j \in\{1, \ldots, n\}$ and $k \in\{1, \ldots, m\}$.

Definition $7 A$ consensus measure on $\mathcal{R}(X)^{m}$ is a mapping

$$
\mathcal{M}: \mathcal{R}(X)^{m} \times \mathcal{P}_{2}(V) \longrightarrow[0,1]
$$

that satisfies the following conditions:

(1) Unanimity. For all $\boldsymbol{R} \in \mathcal{R}(X)^{m}$ and $I \in \mathcal{P}_{2}(V)$, it holds

$$
\mathcal{M}(\boldsymbol{R}, I)=1 \Leftrightarrow\left(R_{i}=R_{j} \text { and } A_{i}=A_{j}, \text { for all } v_{i}, v_{j} \in I\right) .
$$

(2) Anonymity. For all permutation $\pi$ on $\{1, \ldots, m\}, \boldsymbol{R} \in \mathcal{R}(X)^{m}$ and $I \in \mathcal{P}_{2}(V)$, it holds

$$
\mathcal{M}\left(\boldsymbol{R}_{\pi}, I_{\pi}\right)=\mathcal{M}(\boldsymbol{R}, I)
$$

(3) Neutrality. For all permutation $\sigma$ on $\{1, \ldots, n\}, \boldsymbol{R} \in \mathcal{R}(X)^{m}$ and $I \in \mathcal{P}_{2}(V)$, it holds

$$
\mathcal{M}\left(\boldsymbol{R}^{\sigma}, I\right)=\mathcal{M}(\boldsymbol{R}, I)
$$

Unanimity means that the maximum consensus in every subset of decision makers is only achieved when all opinions are the same. Anonymity requires symmetry with respect to decision makers and neutrality means symmetry with respect to alternatives.

We now introduce additional properties that a consensus measure may satisfy.

Definition 8 Let $\mathcal{M}: \mathcal{R}(X)^{m} \times \mathcal{P}_{2}(V) \longrightarrow[0,1]$ be a consensus measure.

(1) $\mathcal{M}$ satisfies maximum dissension if for all $\boldsymbol{R} \in \mathcal{R}(X)^{m}$ and $v_{i}, v_{j} \in V$ such that $i \neq j$, it holds

$$
\mathcal{M}\left(\boldsymbol{R},\left\{v_{i}, v_{j}\right\}\right)=0 \Leftrightarrow\left(R_{i}, R_{j} \in L(X), R_{j}=R_{i}^{-1} \text { and } A_{j}=X \backslash A_{i}\right) .
$$

(2) $\mathcal{M}$ is reciprocal if for all $\boldsymbol{R} \in \mathcal{R}(X)^{m}$ and $I \in \mathcal{P}_{2}(V)$, it holds

$$
\mathcal{M}\left(\boldsymbol{R}^{-1}, I\right)=\mathcal{M}(\boldsymbol{R}, I) .
$$

Maximum dissension means that in each subset of two agents ${ }^{3}$, the minimum consensus level is only reached whenever preferences of agents are linear orders,

$\overline{3}$ It is clear that a society reaches the maximum level of consensus when all the opinions are the same. However, in a society with more than two members it is 
each one the inverse of the other, and the good alternatives of each agent are the bad ones of the other. Reciprocity means that if all individual opinions are reversed, then the consensus does not change.

\section{Measuring consensus for preference-approvals}

We now introduce our proposal for measuring consensus in the context of preference-approvals. Analyzing the homogeneity level in such profiles asks for an extension of the standard measures of consensus in the literature because of the enriched informational content. We show that the consensus measure introduced by García-Lapresta and Pérez-Román [14,24] for weak orders is robust to the additional approval information when the metric proposed by Proposition 1 is used as an input.

Definition 9 Given a metric $d: \mathcal{R}(X) \times \mathcal{R}(X) \longrightarrow \mathbb{R}$, the mapping

$$
\mathcal{M}_{d}: \mathcal{R}(X)^{m} \times \mathcal{P}_{2}(V) \longrightarrow[0,1]
$$

is defined by

$$
\mathcal{M}_{d}(\boldsymbol{R}, I)=1-\frac{\sum_{\substack{v_{i}, v_{j} \in I \\
i<j}} d\left(\left(R_{i}, A_{i}\right),\left(R_{j}, A_{j}\right)\right)}{\left(\begin{array}{c}
\# I \\
2
\end{array}\right) \cdot \Delta_{n}}
$$

where

$$
\Delta_{n}=\max \left\{d\left(\left(R_{i}, A_{i}\right),\left(R_{j}, A_{j}\right)\right) \mid\left(R_{i}, A_{i}\right),\left(R_{j}, A_{j}\right) \in \mathcal{R}(X)\right\} .
$$

Note that the numerator of the quotient appearing in the above expression is the sum of all the distances between the preference-approvals of the profile, and the denominator is the number of terms in the numerator's sum multiplied by the maximum distance between preference-approvals. Consequently, that quotient belongs to the unit interval and it measures the disagreement in the profile.

not an obvious issue to determine when there is the minimum level consensus (the maximum level of disagreement). 


\subsection{Some results}

Proposition 3 For every metric $d: \mathcal{R}(X) \times \mathcal{R}(X) \longrightarrow \mathbb{R}, \mathcal{M}_{d}$ satisfies unanimity and anonymity.

Proof: Let $\boldsymbol{R} \in \mathcal{R}(X)^{m}$ and $I \in \mathcal{P}_{2}(V)$.

(1) Unanimity.

$$
\begin{aligned}
& \mathcal{M}_{d}(\boldsymbol{R}, I)=1 \quad \Leftrightarrow \quad \sum_{\substack{v_{i}, v_{j} \in I \\
i<j}} d\left(\left(R_{i}, A_{i}\right),\left(R_{j}, A_{j}\right)\right)=0 \quad \Leftrightarrow \\
& \Leftrightarrow \quad \forall v_{i}, v_{j} \in I d\left(\left(R_{i}, A_{i}\right),\left(R_{j}, A_{j}\right)\right)=0 \quad \Leftrightarrow \\
& \Leftrightarrow \quad \forall v_{i}, v_{j} \in I \quad\left(R_{i}, A_{i}\right)=\left(R_{j}, A_{j}\right) \quad \Leftrightarrow \\
& \Leftrightarrow \quad \forall v_{i}, v_{j} \in I \quad\left(R_{i}=R_{j} \text { and } A_{i}=A_{j}\right) .
\end{aligned}
$$

(2) Anonymity. Let $\pi$ be a permutation on $\{1, \ldots, m\}$.

$$
\begin{aligned}
& \sum_{\substack{v_{i}, v_{j} \in I_{\pi} \\
i<j}} d\left(\left(R_{\pi(i)}, A_{\pi(i)}\right),\left(R_{\pi(j)}, A_{\pi(j)}\right)\right)= \\
= & \sum_{\substack{v_{\pi(i)}, v_{\pi(j)} \in I \\
\pi(i)<\pi(j)}} d\left(\left(R_{\pi(i)}, A_{\pi(i)}\right),\left(R_{\pi(j)}, A_{\pi(j)}\right)\right)= \\
= & \sum_{\substack{v_{i}, v_{j} \in I \\
i<j}} d\left(\left(R_{i}, A_{i}\right),\left(R_{j}, A_{j}\right)\right) .
\end{aligned}
$$

Thus, $\mathcal{M}_{d}\left(\boldsymbol{R}_{\pi}, I_{\pi}\right)=\mathcal{M}_{d}(\boldsymbol{R}, I)$.

If $\mathcal{M}_{d}$ verifies neutrality, then we say that $\mathcal{M}_{d}$ is the consensus measure associated with $d$.

Proposition 4 If $d: \mathcal{R}(X) \times \mathcal{R}(X) \longrightarrow \mathbb{R}$ is a neutral metric, then $\mathcal{M}_{d}$ is a consensus measure.

Proof: By Proposition $3, \mathcal{M}_{d}$ satisfies unanimity and anonymity. Obviously, if $d$ is neutral, then $\mathcal{M}_{d}$ verifies neutrality and thus $\mathcal{M}_{d}$ is a consensus measure.

Theorem 1 For every $\lambda \in(0,1), \mathcal{M}_{d_{\lambda}}$ is a consensus measure that satisfies maximum dissension and reciprocity.

Proof: By Proposition $4, \mathcal{M}_{d_{\lambda}}$ is a consensus measure. 
(1) Maximum dissension. First, note that $\mathcal{M}_{d_{\lambda}}\left(\boldsymbol{R},\left\{v_{i}, v_{j}\right\}\right)=0$ if and only if $d_{\lambda}\left(\left(R_{i}, A_{i}\right),\left(R_{j}, A_{j}\right)\right)$ is the maximum. This is equivalent to that $d_{R}\left(\left(R_{i}, A_{i}\right),\left(R_{j}, A_{j}\right)\right)$ is the maximum and $d_{A}\left(\left(R_{i}, A_{i}\right),\left(R_{j}, A_{j}\right)\right)$ is the maximum. By Proposition $1, d_{\lambda}\left(\left(R_{i}, A_{i}\right),\left(R_{j}, A_{j}\right)\right)$ is the maximum if and only if $\left(R_{1}, R_{2}\right) \in$ $L(X), R_{2}=R_{1}^{-1}$ and $A_{2}=X \backslash A_{1}$.

(2) Reciprocity. Given $\left(R_{1}, A_{1}\right),\left(R_{2}, A_{2}\right) \in \mathcal{R}(X)$, we only need to prove:

(a) $d_{R}\left(R_{1}, R_{2}\right)=d_{R}\left(R_{1}^{-1}, R_{2}^{-1}\right)$ (see García-Lapresta and Pérez-Román $[14])$.

(b) $d_{H}\left(A_{1}, A_{2}\right)=d_{H}\left(A_{1}^{-1}, A_{2}^{-1}\right)$ :

$$
\begin{aligned}
& d_{H}\left(A_{1}, A_{2}\right)= \\
& =\#\left(\left(A_{1} \cup A_{2}\right) \backslash\left(A_{1} \cap A_{2}\right)\right)= \\
& =\#\left(\left(A_{1} \cup A_{2}\right) \cap\left(A_{1} \cap A_{2}\right)^{-1}\right)= \\
& =\#\left(\left(A_{1} \cup A_{2}\right) \cap\left(A_{1}^{-1} \cup A_{2}^{-1}\right)\right)= \\
& =\#\left(\left(A_{1}^{-1} \cup A_{2}^{-1}\right) \cap\left(A_{1}^{-1} \cap A_{2}^{-1}\right)^{-1}\right)= \\
& =\#\left(\left(A_{1}^{-1} \cup A_{2}^{-1}\right) \backslash\left(A_{1}^{-1} \cap A_{2}^{-1}\right)\right)= \\
& =d_{H}\left(A_{1}^{-1}, A_{2}^{-1}\right) .
\end{aligned}
$$

Taking into account (a) and (b), we have

$$
d_{\lambda}\left(\left(R_{1}, A_{1}\right),\left(R_{2}, A_{2}\right)\right)=d_{\lambda}\left(\left(R_{1}, A_{1}\right)^{-1},\left(R_{2}, A_{2}\right)^{-1}\right) .
$$

Thus, $\mathcal{M}_{d_{\lambda}}\left(\boldsymbol{R}^{-1}, I\right)=\mathcal{M}_{d_{\lambda}}(\boldsymbol{R}, I)$.

\subsection{Illustrative example}

Example 4 Consider again the preference-approvals in Example 2: 


\begin{tabular}{|c|c|c|c|}
\hline$\left(R_{1}, A_{1}\right)$ & $\left(R_{2}, A_{2}\right)$ & $\left(R_{3}, A_{3}\right)$ & $\left(R_{4}, A_{4}\right)$ \\
\hline$x_{1}$ & $x_{2}$ & $x_{1}$ & $x_{3}$ \\
\hline$x_{2}$ & $x_{1}$ & $x_{2}$ & $x_{2}$ \\
\hline$x_{3} x_{4}$ & $x_{3} x_{4}$ & $x_{3} x_{4}$ & $x_{1} x_{4}$ \\
\hline
\end{tabular}

In the following table we illustrate the level of consensus reached in some representative subsets of agents for three values of $\lambda$ :

\begin{tabular}{llll} 
& $\lambda=0.25$ & $\lambda=0.5$ & $\lambda=0.75$ \\
\hline $\mathcal{M}_{d_{\lambda}}\left(\boldsymbol{R},\left\{v_{1}, v_{2}\right\}\right)$ & 0.95833 & 0.91666 & 0.875 \\
\hline $\mathcal{M}_{d_{\lambda}}\left(\boldsymbol{R},\left\{v_{1}, v_{3}\right\}\right)$ & 0.8125 & 0.875 & 0.9375 \\
\hline $\mathcal{M}_{d_{\lambda}}\left(\boldsymbol{R},\left\{v_{3}, v_{4}\right\}\right)$ & 0.64583 & 0.54167 & 0.4375 \\
\hline $\mathcal{M}_{d_{\lambda}}\left(\boldsymbol{R},\left\{v_{1}, v_{2}, v_{3}, v_{4}\right\}\right)$ & 0.69097 & 0.67361 & 0.65625 \\
\hline
\end{tabular}

In the first row the level of consensus is measured for the first two agents. Recall that $\lambda$ is the coefficient for $d_{R}$. Since these agents only disagree on the orderings, an increase in $\lambda$ puts more emphasis for that disagreement and leads to a decrease in the level of consensus. On the contrary, the first and the third agents totally agree on orderings but they disagree on the set of acceptable alternatives. Hence, we can see in the second row that the level of consensus increases when $\lambda$ increases as the importance of that agreement increases (simultaneously, disagreement on the set of acceptable alternatives becomes less important since $1-\lambda$ decreases). In the third row we focus on the third and fourth agents. These agents have disagreement on orderings and on acceptable alternatives at the same time, so they reach the minimum level of consensus for all considered cases so far. Note that the level of consensus decreases for these two agents when the weight of $d_{R}$ increases. Finally, in the last row consensus level is measured for the full profile. We see that as $\lambda$ increases, the level of consensus in the profile decreases. According to these results we conclude that for this profile, individuals have more agreement on which alternatives are socially acceptable than the ordering of those alternatives.

\subsection{Replications}

In some collective decision procedures, especially for the multi-rounded voting systems, analyzing the preference-updating schemes can be useful for the 
moderator to see the changes in the level of consensus. In particular, coalition formations can lead to the occurrence of the same preference as many as the number of the agents in a coalition. Hence, analyzing homogeneity for a given set of preferences when there are some replications of preferences has its own interest. Having this motivation, we consider a metric $d: \mathcal{R}(X) \times \mathcal{R}(X) \longrightarrow \mathbb{R}$ and the associated consensus measure $\mathcal{M}_{d}: \mathcal{R}(X)^{m} \times \mathcal{P}_{2}(V) \longrightarrow[0,1]$. For each $t \in \mathbb{N}$, it is possible to extend $\mathcal{M}_{d}$ to $t$ replicas of profiles of $\mathcal{R}(X)^{m}$ and subsets of $V$ :

$$
\mathcal{M}_{d}^{t}: \mathcal{R}(X)^{t m} \times \mathcal{P}_{2}(t V) \longrightarrow[0,1]
$$

Thus, $\mathcal{M}_{d}^{t}(t \boldsymbol{R}, t I) \in[0,1]$ measures the consensus in the multiset of agents ${ }^{4}$ $t I=I \uplus \cdots \uplus \uplus U$ generated by $t$ replicas of $I$ for the profile generated by $t$ replicas of $\boldsymbol{R} \in \mathcal{R}(X)^{m}, t \boldsymbol{R}=\left(\boldsymbol{R}, .{ }^{t} ., \boldsymbol{R}\right) \in \mathcal{R}(X)^{t m}$.

Proposition 5 Let $d: \mathcal{R}(X) \times \mathcal{R}(X) \longrightarrow \mathbb{R}$ be a metric. For each profile of two preference-approvals $\boldsymbol{R}=\left(\left(R_{1}, A_{1}\right),\left(R_{2}, A_{2}\right)\right) \in \mathcal{R}(X)^{2}$ such that $d\left(\left(R_{1}, A_{1}\right),\left(R_{2}, A_{2}\right)\right)=\delta$ and every $t \in \mathbb{N}$, it holds:

$$
\mathcal{M}_{d}^{t}(t \boldsymbol{R}, t I)=1-\frac{t \cdot \delta}{(2 t-1) \cdot \Delta_{n}},
$$

where $\Delta_{n}=\max \left\{d\left(\left(R_{i}, A_{i}\right),\left(R_{j}, A_{j}\right)\right) \mid\left(R_{i}, A_{i}\right),\left(R_{j}, A_{j}\right) \in \mathcal{R}(X)\right\}$.

Proof: Consider $\boldsymbol{R}=\left(\left(R_{1}, A_{1}\right),\left(R_{2}, A_{2}\right)\right) \in \mathcal{R}(X)^{2}$ with $d\left(\left(R_{1}, A_{1}\right),\left(R_{2}, A_{2}\right)\right)=$ $\delta$ and $I=\left\{v_{1}, v_{2}\right\}$. Given $t \in \mathbb{N}, t \boldsymbol{R}=\left(\left(R_{1}, A_{1}\right), \ldots,\left(R_{2 t}, A_{2 t}\right)\right)$, where $\left(R_{2 k-1}, A_{2 k-1}\right)=\left(R_{1}, A_{1}\right)$ and $\left(R_{2 k}, A_{2 k}\right)=\left(R_{2}, A_{2}\right)$, for every $k \in\{1,2, \ldots, t\}$.

$$
\mathcal{M}_{d}^{t}(t \boldsymbol{R}, t I)=1-\frac{\sum_{\substack{v_{i}, v_{j} \in t I \\
i<j}} d\left(\left(R_{i}, A_{i}\right),\left(R_{j}, A_{j}\right)\right)}{\left(\begin{array}{c}
\#(t I) \\
2
\end{array}\right) \cdot \Delta_{n}} .
$$

Since

$$
d\left(\left(R_{i}, A_{i}\right),\left(R_{j}, A_{j}\right)\right)= \begin{cases}0, & \text { if } i, j \text { are both even } \\ 0, & \text { if } i, j \text { are both odd } \\ \delta, & \text { otherwise }\end{cases}
$$

we obtain

\footnotetext{
4 List of agents where each agent occurs as many times as the multiplicity. For instance, $2\left\{v_{1}, v_{2}\right\}=\left\{v_{1}, v_{2}\right\} \uplus\left\{v_{1}, v_{2}\right\}=\left\{v_{1}, v_{2}, v_{1}, v_{2}\right\}$.
} 


$$
\begin{aligned}
& \sum_{\substack{v_{i}, v_{j} \in t I \\
i<j}} d\left(\left(R_{i}, A_{i}\right),\left(R_{j}, A_{j}\right)\right)=\sum_{i=1}^{2 t-1} \sum_{j=i+1}^{2 t} d\left(\left(R_{i}, A_{i}\right),\left(R_{j}, A_{j}\right)\right)= \\
& =\left(\sum_{i=1}^{t} i+\sum_{j=1}^{t-1} j\right) \delta=t^{2} \cdot \delta .
\end{aligned}
$$

On the other hand, we have

$$
\left(\begin{array}{c}
\#(t I) \\
2
\end{array}\right)=\left(\begin{array}{c}
2 t \\
2
\end{array}\right)=2 t^{2}-t
$$

Consequently,

$$
\mathcal{M}_{d}^{t}(t \boldsymbol{R}, t I)=1-\frac{t \cdot \delta}{(2 t-1) \cdot \Delta_{n}}
$$

Remark 4 Under the assumptions of Proposition 5, it holds:

$$
\lim _{t \rightarrow \infty} \mathcal{M}_{d}^{t}(t \boldsymbol{R}, t I)=1-\frac{\delta}{2 \Delta_{n}} .
$$

Particulary, if $R_{1} \in L(X)$ and $\left(R_{2}, A_{2}\right)=\left(R_{1}^{-1}, A_{1}^{-1}\right)$, then:

$$
\lim _{t \rightarrow \infty} \mathcal{M}_{d}^{t}(t \boldsymbol{R}, t I)=\frac{1}{2} .
$$

Note that Remark 4 illustrates a surprising result that the level of consensus (or homogeneity) in a group of individuals cannot be increased by simply replicating their preferences. In fact, as the particular case of a polarized profile suggests, increasing the number of inverse preferences can only lead to a consensus level of $\frac{1}{2}$.

Example 5 Consider $I=\left\{v_{1}, v_{4}\right\}$ in Example 2. Their preference-approvals over four alternatives are:

$$
\begin{array}{ccc}
\frac{\left(R_{1}, A_{1}\right)}{x_{1}} & & \frac{\left(R_{4}, A_{4}\right)}{x_{3}} \\
\frac{x_{2}}{x_{3} x_{4}} & & x_{2} \\
x_{1} x_{4}
\end{array}
$$


In the following table we illustrate the changes in the level of consensus when we replicate the agents $\left\{v_{1}, v_{4}\right\}$ for three values of $\lambda$ :

\begin{tabular}{llll} 
& $\lambda=0.25$ & $\lambda=0.5$ & $\lambda=0.75$ \\
\hline$\delta=d_{\lambda}\left(\left(R_{1}, A_{1}\right),\left(R_{4}, A_{4}\right)\right)$ & 0.54167 & 0.58333 & 0.66250 \\
\hline $\mathcal{M}_{d_{\lambda}}(\boldsymbol{R}, I)$ & 0.458333 & 0.41666 & 0.375 \\
\hline $\mathcal{M}_{d_{\lambda}}(2 \boldsymbol{R}, 2 I)$ & 0.63889 & 0.61111 & 0.58333 \\
\hline $\mathcal{M}_{d_{\lambda}}(5 \boldsymbol{R}, 5 I)$ & 0.69907 & 0.67593 & 0.65278 \\
\hline $\mathcal{M}_{d_{\lambda}}(15 \boldsymbol{R}, 15 I)$ & 0.71983 & 0.69828 & 0.67672 \\
\hline $\mathcal{M}_{d_{\lambda}}(30 \boldsymbol{R}, 30 I)$ & 0.72457 & 0.70339 & 0.68220 \\
\hline \hline $\lim _{t \rightarrow \infty} \mathcal{M}_{d}^{t}(t \boldsymbol{R}, t I)$ & 0.72917 & 0.70833 & 0.68750 \\
\hline
\end{tabular}

The first row shows the distances between these two preference-approvals with respect to three different values of $\lambda$. Consensus levels are illustrated in the second row. Note that when the size of the profile is doubled by cloning the preferences of each agent, as it is shown in the third row, consensus levels are increased for each values of $\lambda$. According to the results in table, we see that as the number of replications are increased the level of consensus also increases as it might be expected. However, our results also show that there exists a limit for increasing the homogeneity level in a group of individuals by simply replicating their preferences.

\section{Concluding remarks}

Many collective decision making problems of voting, matching, bargaining and public goods implicitly use some threshold levels which are naturally described in the preference-approval framework. We explore the problem of measuring consensus in this hybrid informational system by following a distance-based approach. Measuring homogeneity in terms of distances raises the question of how to evaluate the similarity of any two preferences. Although this question has been answered for various types of ordinal rankings like linear or weak orders over alternatives, we are not aware of a formal treatment of this problem for non-standard preferences. Enriching the informational content by approval notion asks for a more sophisticated evaluation of similarity of preferences.

Given any two preference-approvals we propose measuring the concordance of them by convex combinations of normalized Kemeny and Hamming metrics. At this stage, our proposal depends on a priori selection of the coefficients 
( $\lambda$ and $1-\lambda$ ) for Kemeny and Hamming metrics depending on the context of the relevant problem. Due to the relative importance of the disagreement with respect to the orderings of the alternatives or the approval of them, $\lambda$ can be chosen by a moderator or by an aggregation rule. Experimental studies related to the optimal selection of $\lambda$ for different contexts would give more insight for the implementation of this procedure, but this would be the subject of a separate paper.

By deriving a proper metric that takes into account two pieces of information, next we deal with measuring homogeneity according to these two components. We see that the measure of consensus introduced by García-Lapresta and Pérez-Román [14] can be extended for preference-approvals when that measure is based on a metric that satisfies some desirable axioms. Among the interesting results, we show that the degree of homogeneity in a group of individuals cannot be increased by simply replicating their preference-approvals.

For further research, analyzing metrics which can identify correlation between rankings and accepted alternatives in preference-approvals invites interesting questions. Additionally, using weighted distances to measure the discrepancies with respect to the position of the alternatives in the rankings would complement this paper. How to apply our model for truncated preferences on the subsets of a given alternative set arises another appealing question, especially when there is large number of alternatives under consideration.

\section{Acknowledgements}

The authors are grateful to Jorge Alcalde-Unzu, Miguel Ángel Ballester, Ayça Giritligil and Jean Lainé for their suggestions and comments. The financial support of the Spanish Ministerio de Ciencia e Innovación (projects ECO200907332 and ECO2008-03204-E/ECON) and ERDF are gratefully acknowledged. This paper is also an outcome of a project (107K560) supported by the Scientific and Technological Research Council of Turkey for which the authors are grateful as well.

\section{References}

[1] J. Kacprzyk, M. Fedrizzi, A 'human - consistent' degree of consensus based on fuzzy logic with linguistic quantifiers, Mathematical Social Sciences 18 (1989) $275-290$.

[2] W.J. Tastle, M.J. Wierman, Consensus and dissention: A measure of ordinal dispersion, International Journal of Approximate Reasoning 45 (2007) 531-545. 
[3] W.J. Tastle, M.J. Wierman, Corrigendum to: Consensus and dissention: A measure of ordinal dispersion, International Journal of Approximate Reasoning 51 (2010) 364 .

[4] J.G. Kemeny, Mathematics without numbers, Daedalus 88 (1959) 571-591.

[5] G. Beliakov, T. Calvo, S. James, On penalty-based aggregation functions and consensus, in: E. Herrera-Viedma, J.L. García-Lapresta, J. Kacprzyk, H. Nurmi, M. Fedrizzi, S. Zadrózny (Eds.), Consensual Processes, STUDFUZZ 267, Springer-Verlag, Berlin, 2011, pp. 23-40.

[6] L. Susskind, S. McKearnan, J. Thomas-Larmer, The Consensus Building Handbook: A Comprehensive Guide to Reaching Agreement, SAGE Publications, Thousand Oaks, 1999.

[7] D. Straus, T.C. Layton, How to Make Collaboration Work: Powerful Ways to Build Consensus, Solve Problems and Make Decisions, Berret-Koehler Publishers, San Francisco, 2002.

[8] M. Van Den Belt, Mediated Modelling: A System Dynamics Approach to Environmental Consensus Building, Island Press, Washington DC, 2004.

[9] M. Martínez-Panero, Consensus perspectives: Glimpses into theoretical advances and applications, in: E. Herrera-Viedma, J.L. García-Lapresta, J. Kacprzyk, H. Nurmi, M. Fedrizzi, S. Zadrózny (Eds.), Consensual Processes, STUDFUZZ 267, Springer-Verlag, Berlin, 2011, pp. 179-193.

[10] M. Kendall, J.D. Gibbons, Rank Correlation Methods, Oxford University Press, New York, 1990.

[11] W.L. Hays, A note on average tau as a measure of concordance, Journal of the American Statistical Association 55 (1960) 331-341.

[12] J. Alcalde-Unzu, M. Vorsatz, Measuring consensus: Concepts, comparisons, and properties, in: E. Herrera-Viedma, J.L. García-Lapresta, J. Kacprzyk, H. Nurmi, M. Fedrizzi, S. Zadrözny (Eds.), Springer-Verlag, Berlin, 2011, pp. 195211.

[13] R. Bosch, Characterizations of Voting Rules and Consensus Measures, Ph. D. Dissertation, Tilburg University, 2005.

[14] J.L. García-Lapresta, D. Pérez-Román, Measuring consensus in weak orders, in: E. Herrera-Viedma, J.L. García-Lapresta, J. Kacprzyk, H. Nurmi, M. Fedrizzi, S. Zadrózny (Eds.), Springer-Verlag, Berlin, 2011, pp. 213-234.

[15] J.L. García-Lapresta, D. Pérez-Román, Consensus measures generated by weighted Kemeny distances on weak orders, Procceedings of the 10th International Conference on Intelligent Systems Design and Applications, Cairo, 2010 .

[16] S.J. Brams, P.C. Fishburn, Approval Voting, second edition, Springer, New York, 2007. 
[17] M. Balinski, R. Laraki, Majority Judgment: Measuring, Ranking and Electing, The MIT Press, Cambridge MA, 2011.

[18] W.D. Smith, On Balinski and Laraki's Majority Judgement median-based range-like voting scheme, http://rangevoting.org/MedianVrange.html, 2007.

[19] S.J. Brams, M.R. Sanver, Voting systems that combine approval and preference, in: S.J. Brams, W.V. Gherlein, F.S Roberts (Eds.), The Mathematics of Preference, Choice and Order: Essays in Honour of Peter C. Fishburn, Studies in Choice and Welfare, Springer-Verlag, Berlin, 2009, pp. 215-237.

[20] R.W. Hamming, Error detecting and error correcting codes, Bell System Technical Journal 29 (1950) 147-160.

[21] M.M. Deza, E. Deza, Encyclopedia of Distances, Springer-Verlag, Berlin, 2009.

[22] W.D. Cook, M. Kress, L.M. Seiford, A general framework for distance-based consensus in ordinal ranking models, European Journal of Operational Research 96 (1996) 392-397.

[23] D. Eckert, C. Klamler, Distance-based aggregation theory, in: E. HerreraViedma, J.L. García-Lapresta, J. Kacprzyk, H. Nurmi, M. Fedrizzi, S. Zadrózny (Eds.), Springer-Verlag, Berlin, 2011, pp. 3-22.

[24] J.L. García-Lapresta, D. Pérez-Román, Some consensus measures and their applications in group decision making, in: D. Ruan, J. Montero, J. Lu, L. Martínez, P. D'hondt, E.E. Kerre (Eds.), Computational Intelligence in Decision and Control, World Scientific, Singapore, 2008, pp. 611-616. 